\title{
ESTUDO CINÉTICOS DA REDUÇÃO DE PELOTAS DE POEIRA DE ACIARIA LD POR HIDROGÊNIO
}

Eduardo Junca '

Thomaz Augusto Guisard Restivo ${ }^{2}$

Denise Crocce Romano Espinosa '

Jorge Alberto Soares Tenório '

\section{Resumo}

A geração de poeira de aciaria LD é um dos problemas encontrados por empresas do setor siderúrgico. Desta forma, torna-se importante o desenvolvimento de estudos que possam viabilizar, ou pelo menos indicar um caminho para a reutilização deste resíduo. A técnica de redução direta pode ser uma alternativa, uma vez que, produtos ricos em ferro metálicos são obtidos. Assim, este trabalho tem por objetivo estudar a cinética de redução de pelotas feitas com poeira de aciaria LD através de uma mistura contendo hidrogênio e argônio, obtendo assim os mecanismos que estão envolvidos na redução das pelotas e a energia de ativação aparente envolvida em cada etapa. Aplicou-se a técnica conhecida como Forced Stepwise Isothermal Analysis na investigação cinética entre as temperaturas de $500^{\circ} \mathrm{C}$ a $1.100^{\circ} \mathrm{C}$. Os resultados mostraram que a reação de redução ocorreu em três etapas. Na primeira etapa, o mecanismo controlador foi por nucleação com uma energia de ativação de $24 \mathrm{~kJ} / \mathrm{mol}$. Na segunda e terceira etapa, o mecanismo controlar foi por difusão, porém, na segunda etapa, a energia de ativação aparente obtida foi de $54,9 \mathrm{~kJ} / \mathrm{mol}$ enquanto que na terceira etapa foi de $80,7 \mathrm{~kJ} / \mathrm{mol}$. Palavras-chave: Cinética química; Poeira de aciaria LD; Redução direta.

\section{KINETIC STUDY OF REDUCTION OF BASIC OXYGEN FURNCE DUST PELLETS BY HYDROGEN}

\begin{abstract}
\section{INTRODUÇÃO}

A aciaria LD é responsável pela produção de $60 \%$ do total de aço produzido, sendo o segundo maior gerador de resíduos sólidos de uma usina siderúrgica integrada, contribuindo com $27 \%$ do resíduo sólido gerado numa planta. Isto representa um total de 5 a 7 milhões de toneladas de resíduos gerados mundialmente por ano [I-3].
\end{abstract}

The generation of basic oxygen furnace dust is a problem that companies found in the steel industry. In this way, studies are important to obtain, or at least indicate a way to recycle this waste. The direct reduction can be an alternative, once this method produces rich in iron. Thus, the goal of this paper is study the kinetic of reduction of basic oxygen furnace dust pellet using a mixture containing hydrogen and argon. Thereby, the mechanism and apparent activation energy that involve the reduction can be found out. The kinetic analysis was carried out applying the Forced Stepwise Isothermal Analysis method. The temperatures used in the test were between $500^{\circ} \mathrm{C}$ to I, $100^{\circ} \mathrm{C}$. The results showed that reduction occurred in three steps. In the first step, nucleation was the control mechanism. It was found an apparent activation energy about $24 \mathrm{~kJ} / \mathrm{mol}$ in this step. In the second and third stage, the control mechanism was diffusion. In the second step, the apparent activation energy was $54.9 \mathrm{~kJ} / \mathrm{mol}$, while in the third step the apparent activation energy was $80.7 \mathrm{~kJ} / \mathrm{mol}$.

Keywords: Chemical kinetics; Basic oxygen furnace dust; Direct reduction.

Poeira de aciaria LD é composta principalmente por óxidos de ferro, como por exemplo, magnetita e wustita. Fases a base de cálcio também podem ser encontrados, como por exemplo, calcita. A Tabela I apresenta composições típicas de poeira de aciaria LD citadas pela literatura.

\footnotetext{
'Departamento de Engenharia Metalúrgica e de Materiais, Universidade de São Paulo - USP, São Paulo, SP, Brasil. E-mail: eduardojunca@gmail.com; epinosa@usp.br; jtenorio@usp.br

2Universidade de Sorocaba - UNISO, Sorocaba, SP, Brasil.E-mail: thomaz@protolab.com.br
} 
Tabela I. Composição da poeira de aciaria LD [4,5]

\begin{tabular}{ccc}
\hline Componentes & $\begin{array}{c}\text { \% em Peso } \\
\text { (Hay e Rankin) [4] }\end{array}$ & $\begin{array}{c}\text { \% em peso } \\
\text { Scheele e Johansson [5] }\end{array}$ \\
\hline $\mathrm{Total} \mathrm{Fe}_{\mathrm{SiO}}$ & 55,4 & 47,9 \\
$\mathrm{MnO}$ & 2,3 & $\mathrm{I}, 4$ \\
$\mathrm{CaO}$ & $\mathrm{I}, 8$ & 0,3 \\
$\mathrm{MgO}$ & 10,6 & 13,0 \\
$\mathrm{ZnO}$ & 3,7 & 0,5 \\
$\mathrm{~Pb}$ & $\mathrm{I}, 7$ & 6,7 \\
$\mathrm{Na}_{2} \mathrm{O}$ & - & 0,5 \\
\hline
\end{tabular}

Como pode ser observado na Tabela I, o ferro é o principal elemento encontrado na poeira de aciaria LD. No caso dos exemplos citados, foram obtidos teores de ferro total de $55,4 \%$ e $47,9 \%$.

Na redução de óxidos de ferro por redução direta, é possível a obtenção de produtos com elevado teor de ferro metálico, com isso, podendo ser usados em processos de fabricação de aço, como por exemplo, na aciaria elétrica.

O processo de redução direta, baseia-se na reação de redução de um gás redutor, hidrogênio e $\mathrm{CO}$ por exemplo, com um óxido, gerando um produto diferente do reagente inicial. Este processo é um tipo de reação gás-sólido. Desta forma, a Equação I [6] apresenta a equação genérica para reações do tipo gás-sólido.

$$
\mathrm{A}_{(\mathrm{g})}+b \mathrm{~B}_{(\mathrm{s})}=c \mathrm{C}_{(\mathrm{g})}+d \mathrm{D}_{(\mathrm{s})}
$$

Onde $b, c$ e $d$ são os coeficientes estequiométricos da reação.

A reação de redução de $\mathrm{Fe}_{2} \mathrm{O}_{3}$ pode ocorrer das seguintes maneiras [7]:

$\checkmark$ Uma cadeia de reações simples, Equação 2:

$$
\mathrm{Fe}_{2} \mathrm{O}_{3} \rightarrow \mathrm{Fe}_{3} \mathrm{O}_{4} \rightarrow \mathrm{FeO} \rightarrow \mathrm{Fe}
$$

$\checkmark$ Combinações de reações simples e duplas, Equações 3 e 4:

$$
\begin{aligned}
& \mathrm{Fe}_{2} \mathrm{O}_{3} \rightarrow \mathrm{Fe}_{3} \mathrm{O}_{4} \rightarrow \mathrm{Fe} \\
& \mathrm{Fe}_{2} \mathrm{O}_{3} \rightarrow \mathrm{FeO} \rightarrow \mathrm{Fe}
\end{aligned}
$$

Na redução de óxidos de ferro por agentes gasosos, pode-se encontrar diferentes valores de energia de ativação e mecanismos controladores. A variação tanto dos valores de energia de ativação como dos mecanismos controladores estão ligados aos parâmetros utilizados nos ensaios, como por exemplo, material inicial, a natureza do gás redutor, a faixa de temperatura utilizada e impurezas na amostra [8].

Desta forma, o objetivo deste trabalho é realizar a redução direta de pelotas feitas com poeira de aciaria LD através de mistura redutora contendo hidrogênio e argônio, obtendo assim os mecanismos controladores da reação de redução e ao mesmo tempo, obter os valores de energia de ativação aparente para cada etapa envolvida.

\section{MATERIAIS E MÉTODOS}

A caracterização da poeira de aciaria LD utilizada neste trabalho pode ser visualizada em publicações anteriores [9].

A partir da poeira de aciaria LD, foram confeccionadas pelotas. As pelotas foram feitas através de um disco pelotizador. Em seguida, as mesmas foram secas até a temperatura de $700^{\circ} \mathrm{C}$ com o objetivo de decompor $\circ \mathrm{CaCO}_{3}$ que contem na pelota, uma vez que o mesmo pode interferir na análise termogravimétrica. No estudo de Mikhail e Turcotte [I] foi observado que entre $500^{\circ} \mathrm{C}$ a $720^{\circ} \mathrm{C}$ ocorreu a decomposição de carbonato de cálcio da amostra de poeira de aciaria LD.

Após a confecção das pelotas, foi iniciada a etapa de análise térmica utilizando uma termobalança SETARAM. As análises foram efetuadas utilizando uma mistura contendo $90 \%$ de argônio e $10 \%$ de hidrogênio a um fluxo de $200 \mathrm{~mL} /$ minuto, que foi controlado pelo mass flow do equipamento. As análises termogravimétricas deste trabalho foram desenvolvidas com o intuído de aplicar o método descrito por Sorensen [10-12]. Este método é conhecido por Forced Stepwise Isothermal Analysis (FSIA) no qual na programação térmica são descritas várias isotermas em um único ensaio.

Deste modo, optou-se por trabalhar com temperaturas entre $500^{\circ} \mathrm{C}$ a $1.100^{\circ} \mathrm{C}$, com criação de isotermas a cada $50^{\circ} \mathrm{C}$, ou seja, isotermas foram programas nas temperaturas de $500^{\circ} \mathrm{C}, 550^{\circ} \mathrm{C}, 600^{\circ} \mathrm{C}, 650^{\circ} \mathrm{C}, 700^{\circ} \mathrm{C}, 750^{\circ} \mathrm{C}, 800^{\circ} \mathrm{C}$, $850^{\circ} \mathrm{C}, 900^{\circ} \mathrm{C}, 950^{\circ} \mathrm{C}, 1.000^{\circ} \mathrm{C}, 1.050^{\circ} \mathrm{C}$ e $1.100^{\circ} \mathrm{C}$. Cada isoterma teve duração de 15 minutos.

Com os dados obtidos nas análises termogravimétricas (perda de massa neste caso), foi possível determinar o valor da fração reagida, através da Equação 5 [13-|5].

$$
\propto=\frac{m_{0}-m_{T}}{m_{0}-m_{f}}
$$

A fração reagida aparece nas principais funções citadas pela literatura que envolve reações do tipo gás-sólido, que são apresentadas na Tabela 2.

Deste modo, a avaliação cinética foi desenvolvida da seguinte forma:

$\checkmark$ Levantamento da curva dTG para observação de possíveis mudanças na taxa de reação.

$\checkmark$ Avaliação dos fatores de correlação $\left(R^{2}\right)$ das relações $\mathrm{d}(\alpha) / \mathrm{dt} \times \mathrm{f}(\alpha)$ das funções apresentadas na Tabela 2 para pré-escolha de possíveis funções controladoras. Funções que apresentam maior linearidade são possíveis controladoras.

$\checkmark$ Levantamento das curvas de Arrhenius para as funções pré-determinadas na etapa anterior. 
Tabela 2. Funções controladoras citadas pela literatura como principais mecanismos de reações do tipo gás-sólido [17,18]

\begin{tabular}{lcc}
\hline \multicolumn{1}{c}{ Mecanismo } & Símbolo & $\mathbf{f ( \alpha )}$ \\
\hline Reação controlada por limite de fases & R2 & $(I-\propto)^{1 / 2}$ \\
Reação controlada por limite de fases & R3 & $(I-\propto)^{1 / 3}$ \\
Difusão unidimensional & DI & $\frac{1}{\propto}$ \\
Difusão bidimensional & D2 & $\frac{1}{-\ln (I-\propto)}$ \\
Difusão tridimensional (equação de Jander) & D3 & $\frac{(I-\propto)^{2 / 3}}{I-(I-\propto)^{1 / 3}}$ \\
Difusão tridimensional (equação de Ginstling-Brounshtein) & D4 & $\frac{\left[(I-\propto)^{-1 / 3}-I\right]}{(I-\propto)[-\ln (I-\propto)]^{1 / 2}}$ \\
Crescimento de núcleo bidimensional & A2 & A3 \\
\hline
\end{tabular}

$\checkmark$ Comparação dos fatores de correlação $\left(R^{2}\right)$ obtidas nas curvas de Arrhenius para cada função.

$\checkmark$ Comparação dos resultados encontrados neste trabalho com o que a literatura sugere.

Este método de análise foi empregado em uma tese de doutorado na Universidade de São Paulo [16].

Critérios também foram adotados para interpretação dos dados obtidos na análise termogravimétrica. Deste modo, - primeiro e último patamar em que a reação ocorreu foi descartado. No primeiro patamar da análise de redução foi observado que a reação ainda não havia iniciado. No caso do último patamar, foi observado que as reações de redução se encerravam durante o mesmo, portando, não sendo possível realizar os cálculos cinéticos com os dados de perda de massa obtidos.

\section{RESULTADOS E DISCUSSÃO}

\section{I Análise Cinética}

Inicialmente, foi levantada a curva de perda de massa para a redução da pelota de poeira de aciaria LD reduzida com mistura contendo hidrogênio e argônio (Figura I).

Foi observado que a perda de massa total ao fim da reação foi de $26 \%$. Além disso, foi observado que a reação de redução foi finalizada à $1.050^{\circ} \mathrm{C}$, fato que pode ser comprovado pela linearidade da curva de perda de massa após esta temperatura. Em seguida, foi levantada a curva com a primeira derivada da perda de massa (Figura 2). Foram analisados os fatores de correlação das relações $d(\alpha) / d t x$ $f(\alpha)$ (Tabela 3) para as funções das apresentadas na Tabela 2.

Pela Figura 2 é observado que a redução ocorreu em três etapas: $550^{\circ} \mathrm{C}$ a $650^{\circ} \mathrm{C}$ (região A), $700^{\circ} \mathrm{C}$ a $850^{\circ} \mathrm{C}$ (região B) e $900^{\circ} \mathrm{C}$ a $1.000^{\circ} \mathrm{C}$ (região C).

Através da análise dos fatores de correlação apresentados na Tabela 3 é possível observar que para a primeira etapa da redução, as funções A2, A3, DI, D2, D3 e R3 parecem ser as que apresentam maior linearidade. Desta forma, podendo ser candidatas a funções controladoras.

Para as outras duas etapas, $700^{\circ} \mathrm{C}$ a $850^{\circ} \mathrm{Ce} 900^{\circ} \mathrm{C}$ a $1.000^{\circ} \mathrm{C}$, foi observado na Tabela 3 que as funções D2, D3 e D4 aparecem como candidatas a funções controladoras.

A próxima etapa foi a construção das curvas de Arrhenius para todas as funções citadas como possíveis controladoras, ou seja, A2, A3, DI, D2, D3, D4 e R3 (Figura 3).

Como pode ser visto na Figura 3 , das funções pré-escolhidas como possíveis controladoras (A2, $A 3$, DI, D2, D3 e R3), a curva de Arrhenius que melhor se apresentou foi a descrita pela função $A 2$, apresentando um fator de correlação de 0,99 . Portanto, sendo esta função a provável controladora na primeira etapa de redução $\left(550^{\circ} \mathrm{C}\right.$ a $650^{\circ} \mathrm{C}$ ). Esta função implica em uma energia de ativação aparente de $24 \mathrm{~kJ} / \mathrm{mol}$.

Lin et al. [19] encontrou que para a redução de $\mathrm{Fe}_{3} \mathrm{O}_{4}$ para $\mathrm{Fe}$, a mesma função controladora foi obtida. Rao [20] também reportou que a etapa inicial de redução de óxidos de ferro é a nucleação. A nucleação é formada sobre a superfície exposta da partícula. A redução envolve a formação de um núcleo de ferro e seu crescimento sobre a fase de óxido de ferro. $O$ fenômeno de nucleação 


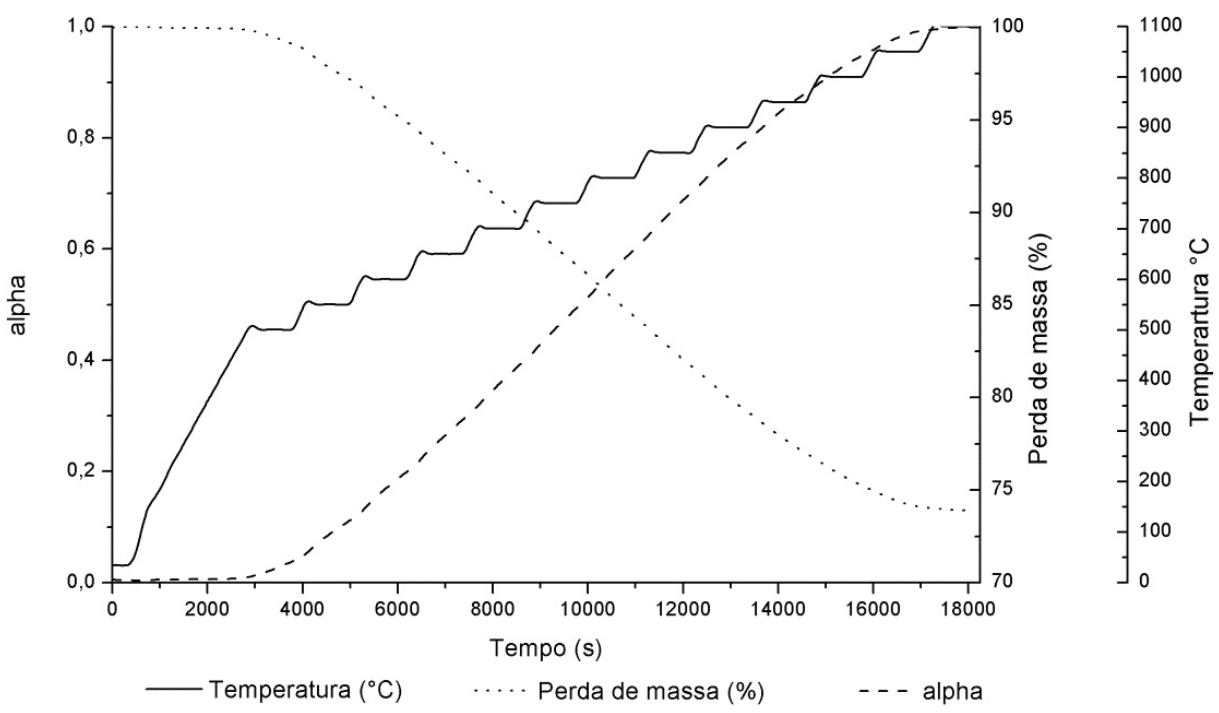

Figura I. Análise termogravimétrica obtida na redução da pelota de poeira de aciaria LD com mistura contendo hidrogênio.

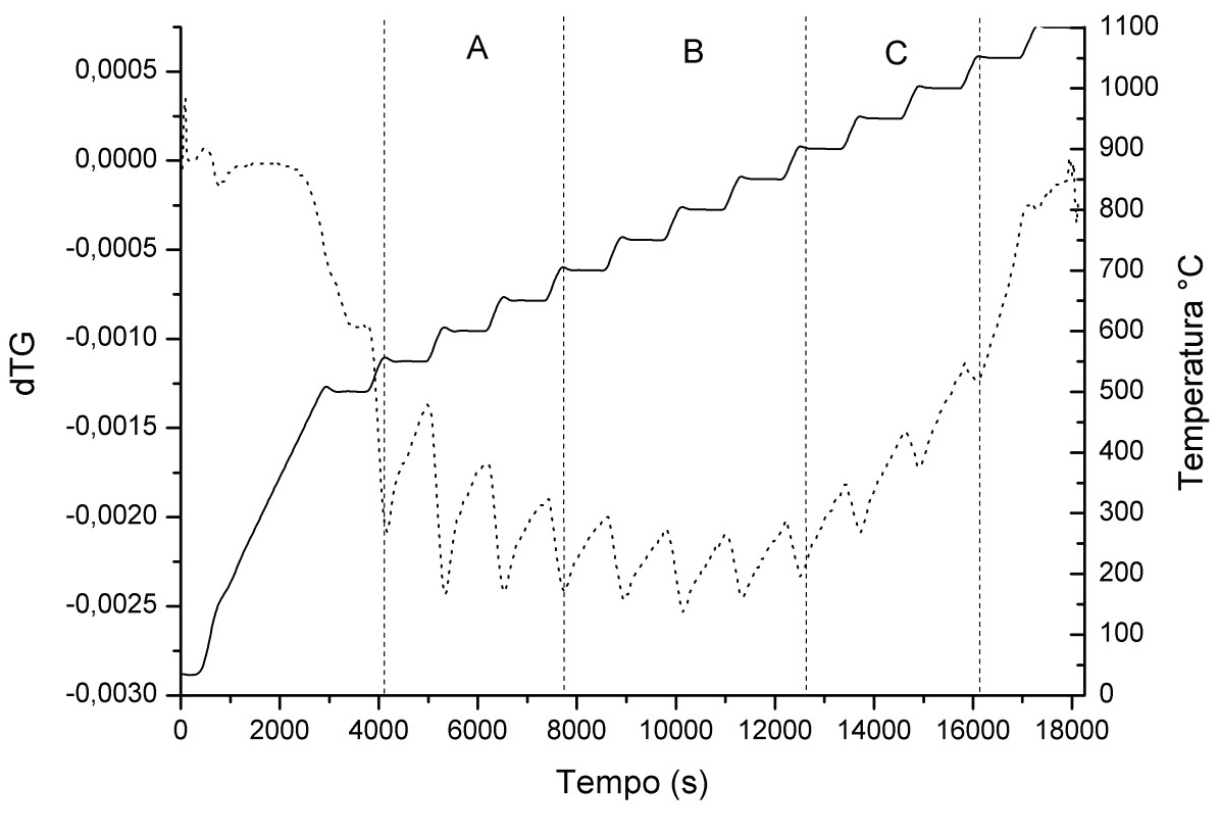

Temperatura ${ }^{\circ} \mathrm{C}$

dTG $200 \mathrm{~mL} / \mathrm{min}$.

Figura 2. Curva dTG da análise termogravimétrica obtida na Figura I.

Tabela 3. Coeficientes de correlação obtidos pela relação $d(\alpha) / d t \times f(\alpha)$ para as funções citadas na Tabela 2

\begin{tabular}{|c|c|c|c|c|c|c|c|c|}
\hline Temperatura & $\mathbf{A 2}$ & A3 & DI & D2 & D3 & D4 & $\mathbf{R 2}$ & $\mathbf{R 3}$ \\
\hline 550 & 0,99 & 0,99 & 0,99 & 0,98 & 0,98 & 0,98 & 0,98 & 0,99 \\
\hline 600 & 0,95 & 0,95 & 0,95 & 0,97 & 0,97 & 0,93 & 0,93 & 0,95 \\
\hline 650 & 0,97 & 0,97 & 0,97 & 0,98 & 0,98 & 0,96 & 0,95 & 0,97 \\
\hline 700 & 0,99 & 0,99 & 0,99 & 0,99 & 0,99 & 0,98 & 0,97 & 0,99 \\
\hline 750 & 0,93 & 0,93 & 0,93 & 0,99 & 0,99 & 0,98 & 0,98 & 0,93 \\
\hline 800 & 0,94 & 0,94 & 0,94 & 0,99 & 0,99 & 0,98 & 0,98 & 0,94 \\
\hline 850 & 0,98 & 0,98 & 0,98 & 0,99 & 0,99 & 0,99 & 0,99 & 0,98 \\
\hline 900 & 0,98 & 0,98 & 0,98 & 0,99 & 0,99 & 0,99 & 0,99 & 0,98 \\
\hline 950 & 0,99 & 0,99 & 0,99 & 0,99 & 0,99 & 0,99 & 0,99 & 0,99 \\
\hline 1.000 & 0,99 & 0,99 & 0,99 & 0,99 & 0,99 & 0,99 & 0,99 & 0,99 \\
\hline
\end{tabular}




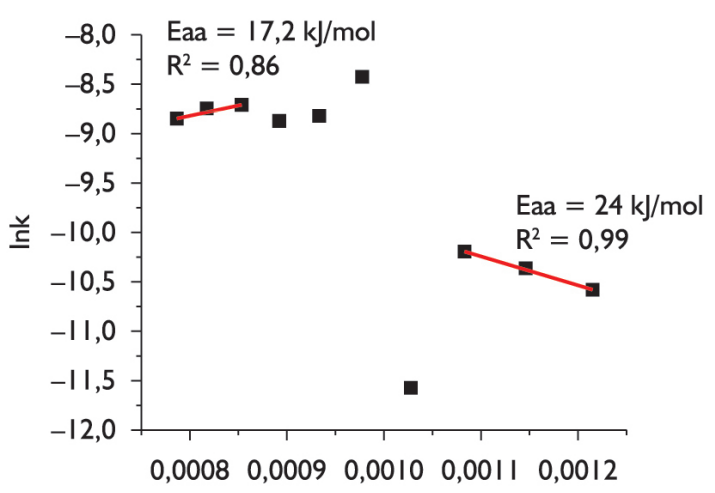

$I / T$
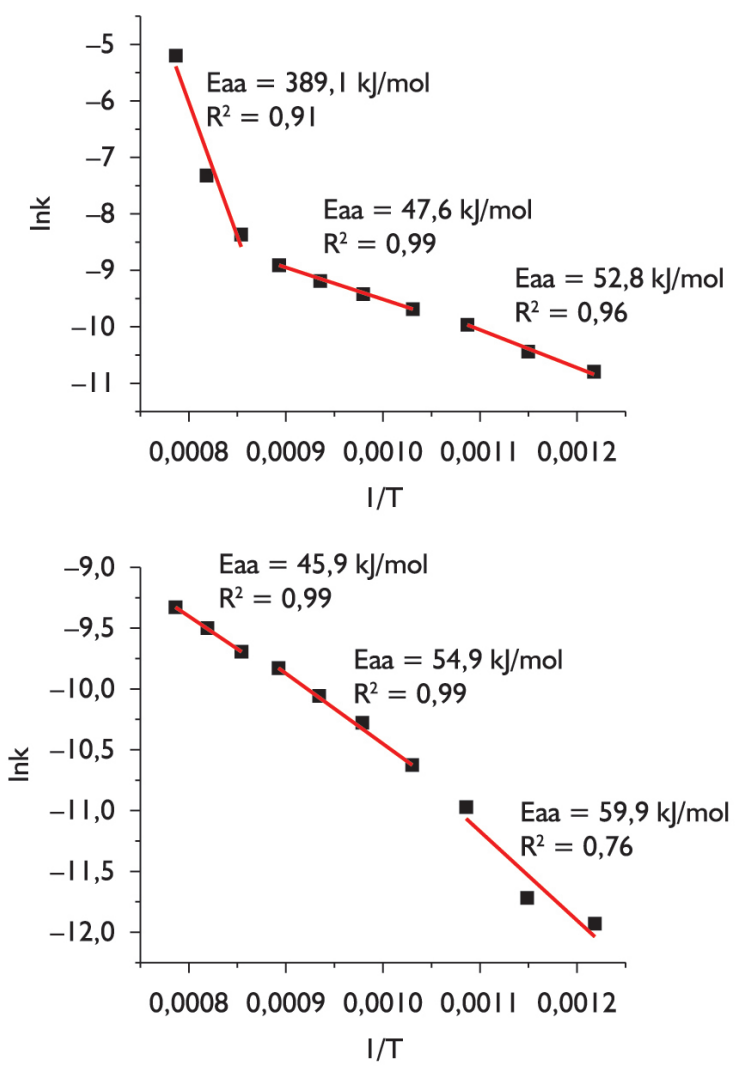

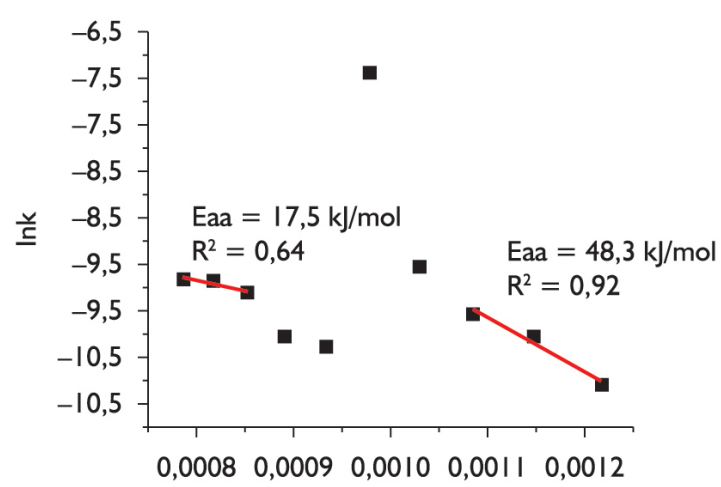

$\mathrm{I} / \mathrm{T}$
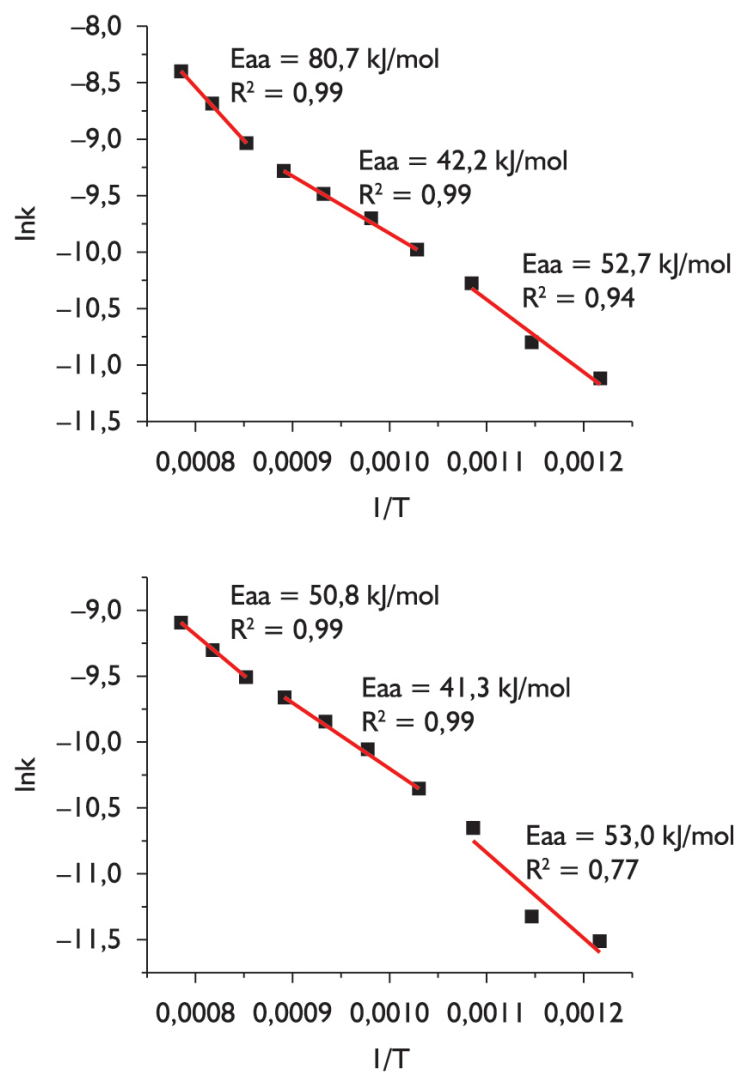

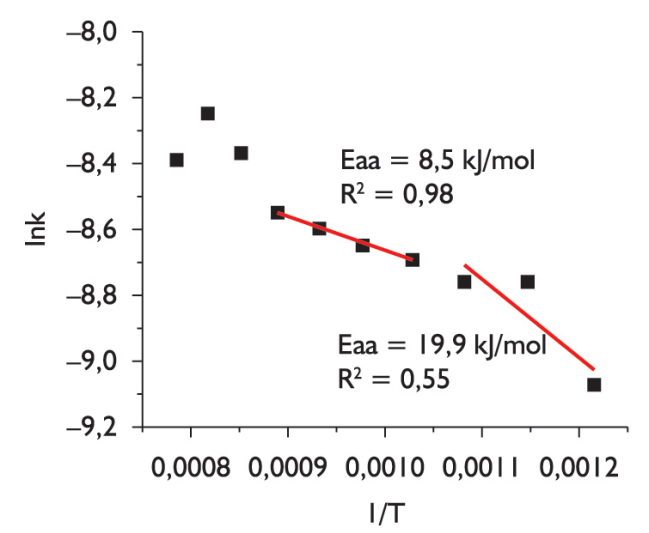

Figura 3. Curvas de Arrhenius aplicando as funções A2, A3, DI, D2, D3 e R3 apresentadas na Tabela 2. 
e crescimento na redução de óxidos de ferro tem sido reportado por outros pesquisadores, tais como Themelis e Gauvin [21], Hayes [22], El-Rahaiby e Rao [23].

Entre $700^{\circ} \mathrm{C}$ a $850^{\circ} \mathrm{C}$, como descrito anteriormente, apenas as funções D2, D3 e D4 aparecem como possíveis controladoras. As curvas de Arrhenius para estas funções mostraram que elas apresentam o mesmo fator de correlação $(0,99)$, portanto, muito provavelmente apresentando um controle misto nesta etapa. Porém, a energia de ativação aparente predominante nesta etapa é de $54,9 \mathrm{~kJ} / \mathrm{mol}$.

A função D3 é citada como uma equação onde a reação ocorrer igualmente em todas as partes das partículas. Entretanto, pode ocorre um decréscimo na taxa de reação com o prosseguimento da reação. Este fato se deve a formação

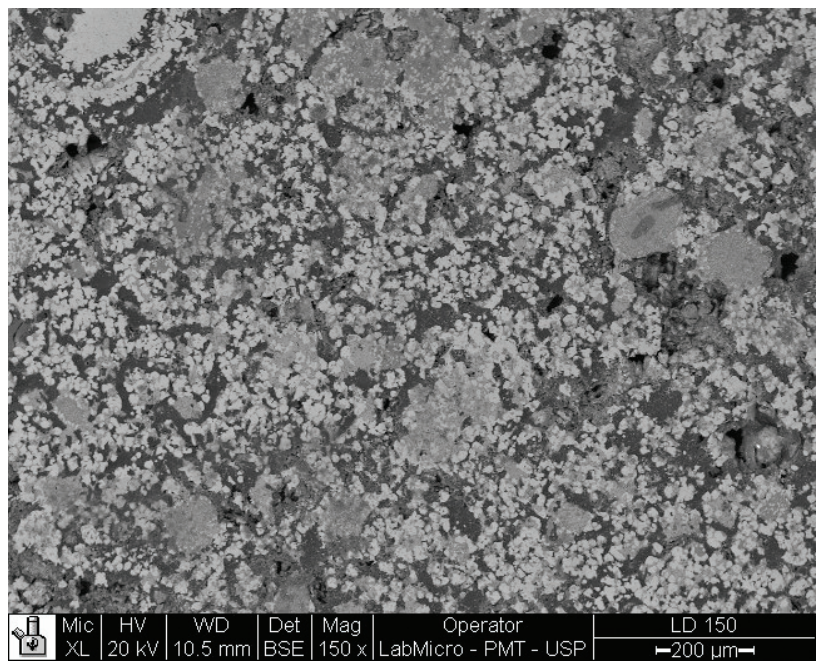

Figura 4. Imagem obtida por microscópio eletrônico de varredura da pelota de poeira de aciaria LD reduzida até $700^{\circ} \mathrm{C}$. e crescimento de uma camada ao redor da partícula que dificulta a continuidade da reação [24].

De acordo com Pineau et al. [25], para redução de $\mathrm{Fe}_{3} \mathrm{O}_{4}$ em temperaturas superiores a $650^{\circ} \mathrm{C}$, a difusão é o mecanismo controlador. Além disso, como pode ser visto na Figura 4, a $700^{\circ} \mathrm{C}$, aparentemente, iniciou-se à sinterização das partículas presentes na pelota. Quando este fenômeno ocorre, um decréscimo na porosidade e na área superficial das partículas pode ocorrer $[26,27]$.

Pineau et al. [25], observaram que para a redução de $\mathrm{Fe}_{3} \mathrm{O}_{4}$ por hidrogeno para temperatura acima de $390^{\circ} \mathrm{C}$, ocorreu a sinterização das partículas.

Por fim, para a última etapa de redução (entre $900^{\circ} \mathrm{C}$ a $1.000^{\circ} \mathrm{C}$ ) as funções D2, D3 e D4 também aparecem como possíveis controladoras. Pelas curvas de Arrhenius das três funções apresentadas na Figura 3, foi observado que as três funções apresentam fatores de correlação de 0,99 , portanto, sendo possível um controle misto.

Foram realizadas análise com auxílio de microscópio eletrônico de varredura na pelota reduzida até o patamar de $900^{\circ} \mathrm{C}$, conforme pode ser visto na Figura 5 .

A Figura 5 a mostra que na temperatura de $900^{\circ} \mathrm{C}$ ocorreu a formação de uma camada reagida em volta do núcleo da pelota. Este núcleo, conforme por ser observado na Figura 5b, apresenta uma estrutura sinterizada e densa, - que pode limitar a passagem do gás redutor em direção ao núcleo da pelota [28].

Valores de energia de ativação entre 62,7 a I46,3 kJ/ mol na redução de briquetes de magnetita em temperaturas entre $800^{\circ} \mathrm{C}$ a $1.000^{\circ} \mathrm{C}$ para valores de fração de reação acima de $55 \%$ tem sido encontrado por pesquisadores [25]. Assim, comparando os valores de energia de ativação aparente encontrados neste trabalho e com os valores encontrados pela literatura, o valor de $80,7 \mathrm{~kJ} / \mathrm{mol}$ encontrado pela função D2 é plausível.

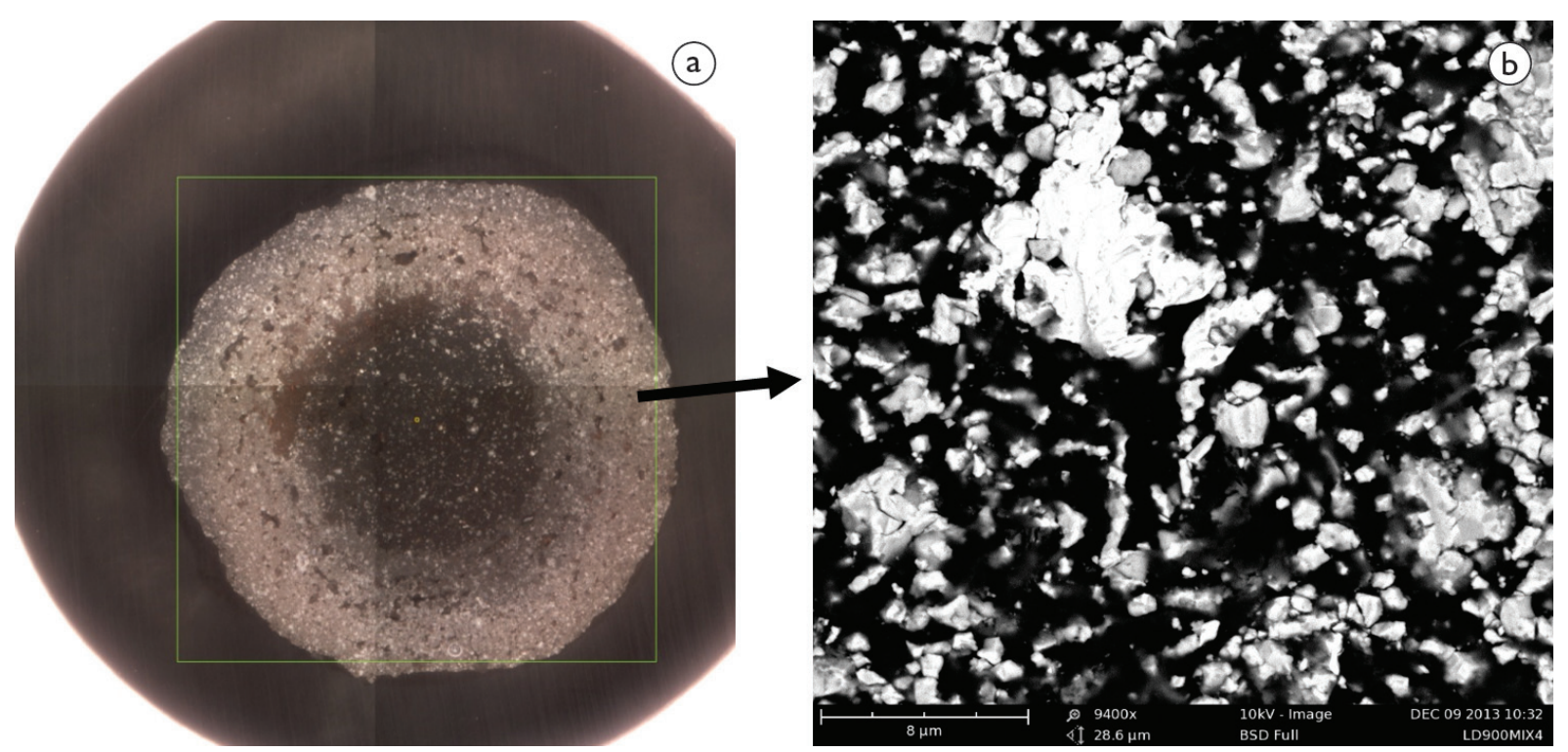

Figura 5. Imagens obtidas da pelota reduzida até a temperatura de $900^{\circ} \mathrm{C}$. 
Além do mais, os autores encontraram a mesma função controladora (D2) quando a temperatura de análise foi superior a $878^{\circ} \mathrm{C}$.

\section{CONCLUSÕES}

A partir dos resultados obtidos neste trabalho, pode-se concluir que as pelotas de poeira de aciaria LD reduzidas com mistura redutora ocorreram em três faixas de temperatura: $550^{\circ} \mathrm{C}$ a $650^{\circ} \mathrm{C}, 700^{\circ} \mathrm{C}$ a $850^{\circ} \mathrm{C}$ e $900^{\circ} \mathrm{C}$ a $1.000^{\circ} \mathrm{C}$. A primeira etapa apresentou uma energia de ativação de $24 \mathrm{~kJ} / \mathrm{mol}$ seguindo o modelo de crescimento de núcleo bidimensional (A2). No segundo estágio, o controle passou a ser controlado pelos modelos D2, D3 e D4 para a difusão. Houve um aumento da energia de ativação aparente para $54,9 \mathrm{~kJ} / \mathrm{mol}$. A última fase também foi controlada por difusão, seguindo os modelos D3 e D4, porém, havendo um acréscimo na energia de ativação, que nesta etapa foi de $80,7 \mathrm{~kJ} / \mathrm{mol}$.

\section{Agradecimentos}

Os autores agradecem à Universidade de São Paulo, FAPESP projeto I I/5 I638-0, à CAPES e ao CNPq 245470/20 I2-3.

\section{REFERÊNCIAS}

I. Mikhail SA, Turcotte AM. Thermal reduction of steel-making secondary materials I. Basic-oxygen-furnace dust. Thermochimica Acta. 1998;3 I (I-2): I I3-I I 9. http://dx.doi.org/I0. 1016/S0040-603 I (97)00430-9.

2. Kelebek S, Yörük S, Davis B. Characterization of basic oxygen furnace dust and zinc removal by acid leaching. Minerals Engineering. 2004;17(2):285-29I. http://dx.doi.org/10.1016/j.mineng.2003.10.030.

3. Evestedt M, Medvedev A. Model-based slopping warning in the LD steel converter process. Journal of Process Control. 2009;19(6): I000-1010. http://dx.doi.org/10.1016/j.jprocont.2009.01.002.

4. Hay SM, Rankin WJ. Recovery of iron and zinc from blast furnace and basic oxygen furnace dusts: A thermodynamic evaluation. Minerals Engineering. 1994;7(8):985-I00I. http://dx.doi.org/I0.1016/0892-6875(94)90028-0.

5. Scheele JV, Johansson M. XYFINES a new technology for in-plant recycling of dust and sludge in metal production industries, recycling and waste treatment in mineral and metal processing. Technical and Economic Aspects. 2002; I:109-118.

6. Szekely J, Evans JW, Sohn HY. Gas-solid reaction. New York: Academic Press; 1976. Reaction of porous solids; p. 108-175.

7. Bessières J, Bessières $A$, Heizmann JJ. Iron oxide reduction kinetics by hydrogen. International Journal of Hydrogen Energy. 1980;5(6):585-595. http://dx.doi.org/10.1016/0360-3199(80)90037-3.

8. Pineau A, Kanari N, Gaballah I. Kinetics of reduction of iron oxides by H2 Part I: Low temperature reduction of hematite. Thermochimica Acta. 2006;447(I):89-100. http://dx.doi.org/I0.1016/j.tca.2005.10.004.

9. Junca E, Oliveira JR, Espinosa DCR, Tenório JAS. Characterization of dust generated in the BOF converter. In: The Minerals, Metals \& Materials Society. Proceedings og the 14 Ist The Minerals, Metals \& Materials Society - TMS; 2012; Orlando, Estados Unidos da America. TMS; 2012. p. 221-227.

10. Sørensen OT. Quasi-isothermal methods in thermal analysis. Thermochimica Acta. 1981;50(I-3):163- I75. http:// dx.doi.org/10.1016/0040-6031(8I)85052-6.

I I. Nigel JC, Andrew KG. Decomposition Reactions of Solids (An Experiment In Reviewing). Thermochimica Acta. 1984;79:323-370. http://dx.doi.org/10.1016/0040-603 I(84)87I I8-X.

12. Sorensen OT. Thermogavimetric and dilatometric studies using stepwise isothermal analysis and related techniques. Journal of Thermal Analysis. 1992;38(I-2):213-228. http://dx.doi.org/10.1007/BF02 109120.

13. Roduit B, Maciejewski M, Baiker A. Influence of experimental conditions on the kinetic parameters of gas-solid reactions -parametric sensitivity of thermal analysis. Thermochimica Acta. 1996;282-283:101-1 19. http://dx.doi. org/I0.1016/0040-603I(96)02882-I.

14. Piotrowski K, Wiltowskim T, Mondal K, Stonawski L, Szymanski T, Dasgupta D. Simultaneous influence of gas mixture composition and process temperature on $\mathrm{Fe} 2 \mathrm{O} 3$ - $\mathrm{FeO}$ reduction kinetics - neural. Brazilian Journal of Chemical Engineering Network Modeling. 2005;22:419-432.

15. Jung SS, Lee JS. In-Situ Kinetic Study of Hydrogen Reduction of Fe2O3 for the Production of Fe Nanopowder. Materials Transactions. 2009;50(9):2270-2276. http://dx.doi.org//0.2320/matertrans.MRA2008472. 
16. Restivo TAG. Redução carbotérmica de óxidos de urânio assistida por banho solvente [tese de doutorado]. São Paulo: Escola Politécnica, Universidade de São Paulo; 2003.

17. Chen F, Sorensen OT, Meng G, Peng D. Thermal decomposition of $\mathrm{BaC} 2 \mathrm{O} 4.0,5 \mathrm{H} 2 \mathrm{O}$ studied by stepwise isothermal analysis and non-isothermal thermogravimetry. Journal of Thermal Analysis. 1998;53(2):397-4I0. http://dx.doi. org/I0.1023/A:1010124905090.

18. Maqueda LAP, Ortega A, Criado JM. The use of master plots for discriminating the kinetic model of solid state reactions from a single constant-rate thermal analysis (CRTA) experiment. Thermochimica Acta. 1996;277: 165-I 73. http://dx.doi.org/10.1016/0040-6031(95)02746-7.

19. Lin Q, Liu R, Chen N. Kinetics of Direct Reduction of Chrome Iron Ore. Journal of Thermal Analysis and Calorimetry. 1999;58(2):317-322. http://dx.doi.org/I0.1023/A:1010190802394.

20. Rao YK. Mechanism and the intrinsic rate of reduction of metallic oxides. Metallurgical Transactions. B, Process Metallurgy. 1979; I0(2):243-255. http://dx.doi.org// 0.1007/BF02652469.

21. Themelis NJ, Gauvin WH. Mechanism of reduction of iron oxides. Canadian Mining and Metallurgical Bulletin. 1962;55:444-456.

22. Hayes PC. The kinetics of formation of $\mathrm{H} 2 \mathrm{O}$ and $\mathrm{CO} 2$ during iron oxide reduction. Metallurgical Transactions. B, Process Metallurgy. 1979; I0(2):2 II-2 I7. http://dx.doi.org/I0.1007/BF02652465.

23. El-Rahaiby SK, Rao YK. The kinetics of reduction of iron oxides at moderate temperatures. Metallurgical Transactions. B, Process Metallurgy. 1979; I (2):257-269. http://dx.doi.org/I0. I007/BF02652470.

24. Khawam A, Flanagan DR. Solid-state kinetic models: basics and mathematical fundamentals. The Journal of Physical Chemistry B. 2006; I 10(35): I73 I5-17328. http://dx.doi.org/10.102I/jp062746a. PMid:16942065

25. Pineau A, Kanari N, Gaballah I. Kinetics of reduction of iron oxides by H2 Part II. Low temperature reduction of magnetite. Thermochimica Acta. 2007;456(2):75-88. http://dx.doi.org/I0.1016/j.tca.2007.01.014.

26. Weiss B, Sturn J, Voglsam S, Strobl S, Mali H, Winter F, et al. Structural and morphological changes during reduction of hematite to magnetite and wustite in hydrogen rich reduction gases under fluidised bed conditions. Ironmaking \& Steelmaking. 20I I;38(I):65-73. http://dx.doi.org/I0.I 179/0301923I0XI2700328926065.

27. Lin HY, Chen YW, Li C. The mechanism of reduction of iron oxide by hydrogen. Thermochimica Acta. 2003;400(I2):6I-67. http://dx.doi.org/10.1016/S0040-603I(02)00478-I.

28. Szekely J, Evans JW, Sohn HY. Gas-solid reaction. New York: Academic Press; 1976. The elements of gas-solid reaction systems involving sigle particles; p. 8-64.

Recebido em: 22 Jul. 2014

Aceito em: 5 Nov. 2014 